\title{
La muerte de la exclusiva: efectos de trasladar el periodismo a Internet
}

\author{
Javier Mato VeIGA \\ Universitat Illes Balears \\ jmato@cesag.org
}

Recibido: 16 de septiembre de 2013

Aceptado: 12 de febrero de 2014

\begin{abstract}
Resumen
Internet, como nuevo canal, tiene sus particularidades que afectan a sus usuarios. El periodismo, con sus valores creados a lo largo de la historia, tiene que adaptarse a la Red. Después de un primer contacto, los periodistas están ahora percibiendo la dimensión real de los cambios. La inmediatez de Internet, conduce a la profesión a una situación en la que todos conocen lo que están publicando los competidores, restándole valor a las noticias exclusivas y tendiendo a la homogeneidad de contenidos. Esto, a su vez, influye en la idea que los periodistas tienen de su profesión, la cual según la sociología de Bourdieu, supone una poderosa afección a sus valore y cultura. Entre ellos, la clásica figura del periodista como portero que decide qué se publica y qué no, queda eclipsada. Utilizando una metodología etnográfica en tres de las más importantes redacciones de España, este artículo explora estos fenómenos bajo el marco teórico de la sociología de Bourdieu, para descubrir que ciertos valores periodísticos muy arraigados están amenazados y en crisis.
\end{abstract}

Palabras clave: Periodismo online, exclusiva, inmediatez, homogeneidad, periodista-portero

\section{The death of scoops.}

The unexpected consequences of doing journalism online

\begin{abstract}
The Internet as a channel has its own characteristics, affecting those who employ it. Journalism, with its own values shaped along the history, has to adapt to the Net. After a decade and a half of relationship, journalists are now realising the full implications of some characteristics associated to the Net. One the them, immediacy, creates an unexpected situation where every medium know what their competitors are offering, then making scoops or exclusive news worthless, and tending to produce similar contents. This trend, in turn, influences the cultural understanding of the profession, which works as a field in Bourdieu's terms, causing an important alteration of values. Among them, the classical figure of the journalist as a 'gatekeeper', who decides what must be socially known or not, fades. Employing an ethnographical approach to three of the more important Spanish newsrooms, this article explores these phenomena under the theoretical umbrella of Bourdieu's sociology, finding that some traditional and well rooted journalistic values are now under threat.
\end{abstract}

Keywords: Online-journalism, scoop, immediacy, homogeneity, gatekeeper

\section{Referencia normalizada}

MATO VEIGA, Javier (2014): "La muerte de la exclusiva: efectos de trasladar el periodismo a Internet". Estudios sobre el Mensaje Periodístico. Vol. 20, Núm. 1 (enero-junio), págs.: 195-210. Madrid, Servicio de Publicaciones de la Universidad Complutense.

Sumario: 1. Introducción. 2. Fundamentos teóricos. 3. Metodología. 4. Resultados; 4.1. La exclusiva; 4.2. Exclusiva y periodista; 4.3. La 'antiexclusiva'. 5. Nuevos valores. 6. Conclusiones. 7. Referencias bibliográficas.

\section{Introducción}

Como ya sucediera en el tránsito de la prensa escrita a la radio y a la televisión (Jones y Salter, 2012: 4-6), el periodismo ha de acomodarse a lo que implica la adopción de 
Internet como nuevo canal de comunicación' ${ }^{1}$. Tras varios años de familiarización, y una vez superados los obstáculos más obvios y superficiales, los periodistas en los medios digitales empiezan a entender las consecuencias más ocultas de usar la red, y cómo estas afectan sus valores, lo cual altera su visión de la propia profesión. Este estudio se centra en cómo la noticia exclusiva tiende a perder peso mientras que lo no exclusivo tiende a extenderse y afianzarse.

\section{Fundamentos teóricos}

El periodismo ha sido descrito como una práctica social, formada a lo largo de los años, basada en un grupo de personas que crean un producto informativo de consumo masivo, constituido de un tipo particular de historias de nuestras vidas diarias. (Park, 1923: 273; Zelizer, 2004: 13-44; Heinonen, 1999: 11). En la medida en que nuestras sociedades se hacen más complejas, estas prácticas adquieren la importancia de mantener informada a la población de que lo ocurre a su alrededor (Domingo et al, 2009: 329). Entonces, en torno a la figura del "comunicador profesional", se crea un producto que se reparte a los ciudadanos (Carey, 1997/1969: 132-133), y que ha sido visto como un elemento importante en la cohesión de las sociedades modernas, al crear una agenda común y nutrir lo que algunos llaman "comunidades imaginadas" (Anderson, 1993: 36). La radio y la televisión, posteriores a la prensa escrita, introdujeron una aceleración en el ciclo de las noticias, pero no alteraron la esencia del papel social del periodismo.

A mediados de los noventa aparece Internet, un nuevo canal para comunicar. Al comienzo, las empresas informativas lo recibieron con los brazos abiertos: sabían que no podían ir lejos repartiendo los periódicos con furgonetas, en tiempos de la tecnología de la información. Tras algunos experimentos con el fax, el teletexto y algunas herramientas que usaban el teléfono, [en] la segunda mitad de los novena [hubo] un momento de efervescencia en relación con el entorno de la web, y los diarios americanos no fueron una excepción: volcaron recursos humanos, financieros y simbólicos en su actividad no impresa, con una intensidad que no se había visto antes. (Boczkowski, 2004: 51)

Los periódicos abrieron masivamente sus sitios online, al punto de que al final de los noventa sólo unos pocos no tenían versión electrónica. En España ocurrió lo mismo: en 2001, más de ochenta periódicos disponían ya de edición papel y online a la vez (Gómez y Paniagua, 2005, s.p.). Esta presencia, sin embargo, no fue muy productiva en un primer momento. Los periódicos acudieron a la red como estrategia defensiva, para evitar que 'su' negocio fuera invadido por empresas de áreas diferentes, pero sin realmente invertir en ello (Nguyen, 2008; Díaz Noci, 2001, López Hidalgo y Mellado Ruiz, 2006). Así, pues, la mayor parte de los medios se limitaron a volcar en Internet el periódico en papel, añadiendo teletipos de agencia para atender la última hora (Boczkowski, 2004: 52-55). En general eran textos similares, al punto de que

Altheide $(1976,1984,1997,2004)$ habla de una lógica interna de cada plataforma, mientras que Postman (2000: 10-11) se refiere a la existencia de un ecosistema interno en cada plataforma. 
David Sancha, en España, llega a detectar en un periódico la repetición del 100 por ciento de los contenidos (Gasher y Gabriele, 2004; Sancha, 2005: 16-17).

Internet llegó acompañado de nuevas características: la hipertextualidad, la multimedialidad, la interactividad y la inmediatez (Steensen, 2010)². Pero su aplicación a fondo se retrasa hasta entrado este siglo, en el entorno de 2006, según autores como Nguyen $(2008,2010)$. Es entonces cuando se percibe que los periodistas eran, como mínimo, reticentes a incorporar y desarrollar lo que la red permite (Hermida y Thurman, 2008, Robinson, 2009). Entre otras cosas, la multimedialidad generó muchos conflictos internos en las redacciones, (Deuze, 2004), tendencia de la que España no ha quedado al margen (García Avilés y Carvajal, 2008). Aunque publicado en 2010, Boczkowski lleva a cabo en 2005 un estudio de tres periódicos electrónicos argentinos, en los que encuentra y describe el fenónemo de la permanente monitorización mutua, llevada a extremos no conocidos en la profesión periodística con anterioridad (Boczkowski, 2010), consecuencia de la inmediatez que proporciona Internet. Angela Phillips ha alertado de cómo la inmediatez se asocia con la copia de noticias por los competidores, subrayando los graves riesgos para quienes producen contenidos originales, la base del periodismo (Phillips, 2010: 95-97).

La llegada de Internet tiene lugar en el templo del periodismo, las redacciones, donde los valores profesionales son compartidos y trasmitidos de los agentes más experimentados a los más noveles (Robinson, 2011; Couldry \& McCarthy, 2004). Desde la segunda parte del siglo pasado, entrar a una redacción y analizar el comportamiento explícito e implícito de quienes operan allí, ha conformado una escuela de trabajo (Warner, 1970 y 1971; Tuchman, 1972, 1974 y 1978; Molotch and Lester, 1974 y 1975; Schlesinger, 1987, entre otros muchos). Hoy, esta tradición se ha ampliado a la investigación de las conductas del periodismo en relación con la red (Møller-Hartley, 2011, ejemplo reciente), incluyendo también una interesante producción dentro de España (Domingo, 2006, entre otros). Algunos autores incluso llegan a afirmar que sólo con esta técnica se puede profundizar en la comprensión de las nuevas realidades del periodismo (Paterson, 2008: 9-10).

Esta asociación entre espacio e ideología profesional adquiere toda su dimensión cuando se estudia al periodismo como un campo o área dentro de la sociedad, en competencia por un lado con otros campos y, por otro, en lucha interna. Es la visión sociológica de Pierre Bourdieu, que describe primero y analiza después cómo los grupos sociales operan, cómo en sus prácticas diarias crean fórmulas para trabajar, para entender el entorno, para valorar y dar sentido a lo que viven y a lo que ocurre, lo que llama "estructuras estructurantes" (Silva y Browne, 2008, s.p.; Bourdieu, 1993; 1996/1992). Su teoría se orienta a los grupos sociales en general, pero dedica una parte significativa de su trabajo a la producción cultural y específicamente a los pe-

2 Pese a que la inmediatez afecta al periodismo online, no fue siempre descatada. Bob Franklin et al. (2005) en su glosario de términos periodísticos titulado Key Concepts in Journalism Studies, no encuentran un espacio para ella en los 238 términos que registra. El mismo Steensen (2010: 2) dice que hay tres características que afectan de lleno al periodismo en la red: hipertextulidad, interactividad y multimedialidad. 
riodistas, afirmando que ellos configuran un campo, donde cada uno tiene su puesto, y compiten con sus colegas por capital (no sólo económico sino también social, simbólico o cultural). En Sobre la televisión (1998/1996), Bourdieu menciona cómo los periodistas leen diaria y obsesivamente todos los periódicos para controlar su campo, para conocer cómo lo están haciendo sus colegas y, anticipando lo que después Internet exacerbará, con algunos ejemplos franceses, afirma que:

"[este] es uno de los mecanismos que hace que los productos periodísticos sean tan similares. Si Liberation dedica titulares a un cierto evento, Le Monde no puede mantenerse indiferente [...]. Estas pequeñas diferencias, a las cuales los periodistas dan gran importancia, ocultan enormes similitudes" (Bourdieu, 1998/1996: 24).

Bourdieu afirma que muchos comportamientos periodísticos son inexplicables a menos que los consideremos como parte de sus luchas internas por lograr un mejor emplazamiento dentro de su campo. Los periodistas desarrollan un "juego de espejos" (1998/1996: 24) con otros medios, pero también internamente, con sus colegas. El campo periodístico tiene herramientas para que sus integrantes demuestren y midan su adaptación, qué dominio tienen de las reglas internas. Los agentes necesitan diferenciarse ellos mismos, para crearse una identidad en el campo, para conseguir capital. "Para existir en un campo... hay que diferenciarse. [...] El o ella existen en virtud de la diferencia respecto de otros intelectuales. Caer en la indiferenciación [...] significa dejar de existir." (Bourdieu, 2005: 39-40). Es lo que llama el habitus, nacido y arraigado en la práctica diaria.

Una de las herramientas mejor valoradas dentro del campo periodístico para mostrar esa diferencia con los compañeros de la propia redacción y de otras, es ser el primer en dar una noticia, en contar algo original hasta ese momento desconocido. Dodson y Sterling comparan la necesidad periodística de ser original con el deseo del académico de ofrecer información novedosa a la comunidad científica (2012: 5). Aunque no muy estudiado por la Academia, quizás porque "los periodistas y los estudiosos del periodismo ven el concepto de la exclusiva como tan obvio que pocos intentan definir el término" (Salwen, 2001: 152), la noticia exclusiva ha sido entendida como una de las referencias de más importancia en el campo profesional del periodismo. Una especie de mito, al menos desde que Evelyn Waugh, el novelista inglés que trabajó como periodista en un viaje a Africa, escribió la famosa novela "Scoop" (Waugh, 1964), donde parodia hasta dónde los periodistas pueden llegar en su obsesión para obtener exclusivas. Esta novela no hace más que retratar lo que aparece como una referencia en casi todos los medios (Marr, 2004).

La exclusiva es parte del habitus profesional del periodismo, lo cual es significativo porque es una parte de los "procesos estructurales de los que se derivan ataduras y limitaciones" sobre los periodistas, "tales como que sus opciones se encuentran totalmente preconfiguradas" (Bourdieu, 2005: 45).

\section{Metodología}

Enlazando el marco teórico interpretativo con la metodología de investigación, Ida Willig (2013) señala: 
Bourdieu ofrece una estrategia analítica para la investigación de la problemática epistemológica permitiendo saltar la distancia entre estructura y agencia, entre el nivel micro y macro, en una aproximación relacional, constructivista y estructuralista. Esto hace de la perspectiva de campo un arma que se adapta al marco de los estudios etnográficos, [...] porque es más que teoría. (Willig, 2013: 384)

Este trabajo ha sido desarrollado como una investigación etnográfica. Siguiendo una tradición muy sólida de investigación en las redacciones periodísticas (Boyer and Hannerz, 2006: 5-7), recomendada también por los académicos para el nuevo entorno mediático digital (Howard, 2002: 550-551; Cottle, 2007), este trabajo se basa en entrevistas y observación participativa, a lo largo de unas 200 horas de trabajo.

Las redacciones estudiadas han sido la de los dos medios online más importantes de España, así como la agencia de noticias líder, que no ofrece un servicio al público en general. Los dos medios online son El País y su rival, El Mundo. En la fecha de la investigación, la edición digital de El Mundo lideraba el ranking, seguida muy de cerca por la de El País, ambas rozando los 30 millones de usuarios únicos mensuales $(\mathrm{OJD}, 2012)^{3}$. La investigación se llevó a cabo a lo largo de tres semanas, desde el 24 de enero al 11 de febrero de 2011. Las características más relevantes de las tres redaccciones son las siguientes:

A) El Mundo, medio digital líder en España (OJD, 2012). Como proyecto periodístico, El Mundo construyó paulatinamente su espacio en el mercado mediático español: nacido al inicio de los noventa, es hoy el segundo periódico generalista más vendido, detrás de El País. Su edición online lidera el mercado desde que en 2002 su rival, El País, decidiera cobrar por acceder a su página web, medida a la que tuvo que renunciar en 2005, tras perder la casi totalidad de sus lectores (Guallar, 2007: 110111). La plantilla, en su mayor parte, tiene larga experiencia en el digital. Es el caso de Fernando Mas, el subdirector; Sonia Aparicio, la directora de reportajes multimedia o de los editores de noticias de última hora, José Luis Martín y Olalla Novoa ${ }^{4}$.

B) Prácticamente desde el momento de su nacimiento, en 1976, El País ha sido el periódico español más influyente. Fue también un medio muy rentable, a pesar de lo cual recientemente está padeciendo problemas financieros de importancia (Gámir, 2005: 195-196). La edición online estaba operada hasta un año antes de esta investigación, por un equipo de personas ajenos a la redacción principal del periódico e, incluso, a la empresa editora, dado que se trataba de una sociedad diferente que operaba

3 La difusión de los periódicos en papel en España sigue los parámetros de la Oficina Justificadora de la Difusión (OJD). En el caso de la difusión de los digitales, hay criterios encontrados. El Mundo está de alta en OJD, que también certifica estas audiencias, mientras que El País utilizaba Nielsen. En cualquier caso, en las entrevistas que mantuve, en los dos periódicos se aceptaba que El Mundo digital era líder, que El País era segundo y que este último estaba creciendo a mayor velocidad que el primero, por lo que no tardaría tiempo en empatar y quizás superarlo.

4 Fernando Baeta, el director del digital de El Mundo, Mas, Novoa, Martín y Aparicio mantuvieron entrevistas personales con el autor los días 24 a 28 de enero de 2011. 
desde un edificio diferente ${ }^{5}$; así, los periodistas producían un periódico en papel que al día siguiente, por la mañana, encontraban cargado en la red, sin su intervención. Las cosas cambian en 2010, cuando la compañía se centra en la edición digital como prioridad. Como consecuencia, la redacción principal asume la operación digital. En el momento en que se hizo este estudio, el ambiente en la redacción era tenso, dado que la dirección de la empresa, que ya había cerrado otras filiales ${ }^{6}$, acababa de anunciar el despido de 2.500 trabajadores, aunque aún no se sabían los nombres de los afectados.

C) Efe es un mayorista de las noticias, una agencia de noticias propiedad del Estado español; su plantilla, casi sólo formada de periodistas, es de las mejor pagadas del país (Olmos, 1997). Aunque esta agencia de noticias no publica noticias online para el público final, Internet ha cambiado su entorno porque todos sus clientes tienen demandas diferentes y porque la red le afecta como fuente de datos. "Para nosotros, Internet ha sido positivo, tenemos más impactos y más clientes. Nuestra crisis tiene que ver con la economía en general, no con Internet", explica Javier Tovar, el director de noticias para España de $\mathrm{Efe}^{7}$. Efe es la primera agencia de noticias del mundo en castellano. En el momento en que se llevó a cabo esta investigación, empleaba unos mil periodistas en 110 países (Boyd-Barrett and Rantanen, 1996: 15-19, 32)8.

Este trabajo se basa en 24 entrevistas en profundidad con periodistas representantes de todos los niveles de responsabilidad en estas tres empresas informativas.

\section{Resultados}

\subsection{La exclusiva}

Es media mañana en la redacción conjunta del diario El Mundo. Los periodistas que están a cargo de las diferentes secciones están atentos a lo que el presidente de la Academia Española del Cine, Alex de la Iglesia, pueda anunciar, dado que ha trascendido su desacuerdo con el gobierno socialista una vez se ha conocido que el proyecto de ley contra la piratería en Internet sería mucho menos exigente de lo que los cineastas pretenden. La edición online de elmundo.es ya tiene en su portada una noticia destacada sobre el tema, pero la redacción teme que alguna reacción más contundente pueda estar preparándose. El redactor jefe de turno de la edición digital, José Luis Martín, ha recibido reiterados avisos de que siga cuidadosamente este asunto. De pronto, en el ruido homogéneo que produce la redacción, se oye: "De la Iglesia dimite" como presidente de la Academia del Cine. “¿Dónde está eso?” pregunta Martín, mientras parece maldecir su suerte. "En su blog de El Pais", el rival comercial declarado de elmundo.es, con quien se disputa el liderazgo en el mercado español. José Luis abre una aplicación en su pantalla y escribe: "Urgente: De la Iglesia dimite como presidente de la Academia Española del Cine". Una cinta de color rojo con letras blancas,

${ }^{5}$ Al comienzo de 2010, la redacción principal de El País se hizo cargo de la edición online. La empresa filial que se encargaba de ella se dedicó a otros fines y finalmente fue disuelta.

${ }^{6}$ Meses antes el grupo PRISA había cerrado una cadena de noticias 24 horas.

7 Entrevista personal con el autor, el 31 de enero de 2011.

${ }^{8}$ Datos actualizados en entrevista con el autor, por Javier Tovar, director de noticias nacionales de Efe. 
situada debajo de la cabecera del periódico, comienza a rodar en la web, dando la noticia. No ha pasado más de un minuto desde que elpais.com publicara la decisión del cineasta. Aunque nadie lo comprobó, podría incluso haberse dado el caso de que la noticia hubiera aparecido como tal antes en elmundo.es que en elpais.com, dado que si bien este segundo medio aportó el dato, lo hizo como en el blog personal de De la Iglesia, alojado en la web del periódico, pero no en la portada informativa.

Simultáneamente, la sección de Cultura de El Mundo está ya escribiendo una noticia breve con la dimisión y sus razones, mientras un ayudante busca una foto de De la Iglesia. “¿Debo decir que la noticia se publicó en El País?” pregunta Luis Alemany, encargado de escribir lo que ocurrió. "Sí, por supuesto", contesta Martín. En tres minutos, una noticia de algo menos de 300 palabras está en la portada de elmundo.es, citando al blog de elpais.com como su fuente .

La noticia exclusiva de elpais.com sobre la dimisión de De la Iglesia estuvo viva no más de tres minutos. El rival más combativo de El País estaba contando la misma dimisión, quizás hasta con más detalles, apenas unos instantes después de El País y, por supuesto, mucho antes de que la enorme mayoría de los lectores se diera cuenta de qué había ocurrido. La inmediatez asociada con Internet está convirtiendo las noticias exclusivas en éxitos efímeros de los que los lectores no llegan a darse cuenta.

Javier Lascurain, antes redactor de Tribunales en la agencia, hoy coordinador de la edición en $E f e$, explica, no sin cierta añoranza, cómo eran las cosas antes:

"Antes, si otro medio tenía una noticia que no conocías, podías llegar a estar varias horas sin enterarte. Incluso podría darse el caso que te enteraras a la mañana siguiente, leyendo la prensa. Yo hacía tribunales y cuando creía que tenía una noticia [exclusiva], nunca la daba hasta que me aseguraba de que mi fuente no podía ser localizada, que ya se había ido a su casa y entonces los rivales no tenían manera de confirmar si era o no verdad. Hoy no, hoy todo se dice en la red y nosotros en cinco minutos ya sabemos lo que se nos pudo pasar".

Lascurain se pregunta "¿por qué debemos hoy correr por una exclusiva cuando en segundos estará online y todos mis colegas podrán localizar a mi fuente y confirmarla?"

Aunque los periodistas reconocen que la exclusiva pierde importancia, los profesionales aún siguen invirtiendo tiempo y esfuerzo en ella, como prueba de lo arraigada que se encuentra en su cultura, lo asentado que se encuentra este valor y, probablemente, del reconocimiento que aún sigue generando. La exclusiva tiene arraigo en el habitus y es creadora de capital, si utilizamos los términos sociológicos de Bourdieu. Por ejemplo, el redactor en jefe de turno de la edición digital de El Mundo, José Luis Martín, confiesa que pese a que recibe en su pantalla el servicio de pago de las principales agencias de noticias del mundo, con líneas dedicadas, él consulta lo mismo en Twitter porque "me llega todo de 15 a 30 segundos antes. Este es un tiempo precioso de cara a publicar una noticia antes, por eso sigo a las agencias en Twitter". Utilizando el marco interpretativo sociológico de Bourdieu, el comportamiento de Lascurain,

9 Notas del autor, tomadas durante el trabajo de observación participativa, el 25 de enero de 2011. 
procurando que su noticia exclusiva tuviera vida durante bastante tiempo, respondía a la necesidad de demostrarle a sus colegas que él tenía cualidades para obtener material informativo de calidad, lo cual enriquecía su prestigio y, por ende, su posición relativa en el campo.

\subsection{Exclusiva y periodista}

Tanto en El Mundo como en El País, las redacciones del periódico papel y del digital se han fusionado. "La idea de dos redacciones separadas con equipos e intereses diferentes es pasado", señala Fernando Baeta, director de elmundo.es, con cierto orgullo por su logro, sugiriendo que no fue fácil. Pese a esta norma general, cada redactor tiene una función primaria en una de las dos ediciones, lo cual aún genera conflictos. Precisamente, en la madrugada siguiente a esta declaración, un conflicto interno surge cuando un redactor del papel conoce una noticia importante en relación con una disputa entre sindicatos y gobierno, y en lugar de aportarla a la edición digital para su difusión inmediata, la guarda para el papel, pese a que probablemente pasaría un día entero antes de su publicación. "Todavía tenemos problemas" se corrige Baeta a sí mismo, "porque todo el mundo quiere las buenas noticias para sí". Tener una noticia es importante para acumular capital, y cederla a otra área significa dejar que otros se apropien de ella o, más importante, del capital asociado a esa noticia. Por eso, todos los agentes implicados perciben la tremenda dificultad en aplicar los principios que Baeta declara.

El director de la edición digital de El Mundo explica el criterio de su empresa: "las exclusivas son para la edición en papel"; "porque [el periódico papel] tiene que ser diferente para sobrevivir, ofrecer algo especial", dice Baeta, mostrando resignación por dejar pasar noticias que le gustarían para su edición, pero aceptando las reglas. "La edición papel está muriendo y yo no estoy aquí para acelerar esta muerte", indica. (Las exclusivas, se deduce de las palabras del director, serían una inyección de vida o, al menos, así lo perciben los integrantes de la redacción, de acuerdo con su idea sobre lo que genera prestigio.) La norma añade que "en caso de duda [que otro medio pueda adelantarse dando esa misma noticia] entonces va en la edición digital". Y este es el punto de colisión. "No es fácil gestionar este conflicto [de intereses]" señala José Luis Martín, quien añade que en este punto crítico todo depende de las actitudes personales. El mismo se pregunta "¿qué hemos de hacer cuando yo tengo un punto de vista original en relación con una noticia? Si en la edición en papel tienen la misma idea, es para ellos. Y eso es muy frustrante porque nosotros también tenemos ideas, pensamos, encontramos noticias", añade con ironía. "Hace unas semanas encontramos una noticia exclusiva sobre el nacimiento de un partido político en Asturias ${ }^{10}$. Lo sabíamos todo pero lo guardamos para la edición en papel. Sin embargo, esa noche una radio dio todos los datos y así ni el papel ni el digital dimos la exclusiva", cuenta Baeta, cuya detallada narración desvela la importancia otorgada a la exclusiva.

${ }^{10}$ Se trata del partido FAC, liderado por Francisco Alvarez Cascos, antiguo dirigente del Partido Popular. 
El País también vive esta dinámica, que es del campo periodístico, no de las empresas. La norma oficial dice que las noticias se publican de inmediato, lo que equivale a que la edición online, que está permanentemente abierta, es prioritaria. Así lo proclama Javier Casqueiro, el responsable de la sección España y lo ratifica Gumersindo Lafuente, director de la edición online, quien explica que "nosotros producimos noticias las 24 horas del día, todos los días, y las publicamos en el canal más cercano para ello, alguna veces online, algunas en el papel". "Pero nos guardamos algo para la edición en papel", corrige con realismo Luis Doncel, responsable de Economía, reconociendo que los redactores tienen otro criterio ${ }^{11}$. "La mayor parte de los redactores-jefes prefieren ofrecer todo lo que tenemos online. Pero cuando algo es realmente interesante... Lo estudiamos caso por caso. No tenemos una línea de acción clara en este sentido", añade Luis Gualdoni, redactor-jefe de Internacional ${ }^{12}$, confirmando la discrepancia entre la norma y el sentimiento de los profesionales que componen la redacción (lo que es su habitus).

"Aunque para el lector El País hace años que está online, nosotros, los miembros de la redacción, hace apenas un año que estamos en contacto. Ahora recién empezamos a experimentar qué significa tener que escribir varias veces la misma noticia sobre los mismos hechos, para las ediciones digitales y en papel". Luis Doncel desvela que hasta hace un año vivían en la cultura del papel, mientras que hoy, cuando un periodista conoce una noticia, primero tiene que dar los datos básicos para la edición digital, después preparar una ampliación completa para esta edición y, finalmente, concluir con una versión para el papel. Doncel, Gualdoni o Iker Seisdedos (redactor jefe de Cultura) manifiestan su interés para gestionar de la mejor forma los diferentes enfoques y objetivos de cada edición, pero sugieren que este nuevo modelo aún necesita tiempo para solidificarse. "Es como el café turco, aún necesita tiempo para sedimentar", dice Gualdoni.

Para Luis Doncel no hay dudas: la idea de la noticia exclusiva se ha diluido. "Las noticias circulan con tal rapidez, que muchas veces no sabemos quién ha sido el primero en contar algo". Lamentándolo, añade que "conservar una noticia exclusiva para publicar más tarde puede suponer perderla, porque otro colega la puede tener," dejando claro que "perderla" sí le importa, de acuerdo con su código de conducta tradicional. El contraste entre el valor otorgado a la exclusiva en el campo periodístico tradicional y la nueva realidad emergente no puede ser más claro. La práctica de siempre, el habitus, formado a lo largo de años, colisiona hoy con una nueva realidad, la derivada de Internet, que no es fácil de aceptar.

\subsection{La 'antiexclusiva'}

Borja Echeverría es el responsable de elpaís.es. Echeverría literalmente corre por la redacción con su i-Pad en la mano. Llega a la mesa central y pregunta por qué tal o cual noticia no está en su edición. Echeverría está constantemente mirando las páginas de los competidores para que no se le escape nada. "En el pasado" explica Eche-

${ }^{11}$ Entrevista personal con el autor, el 10 de febrero de 2011.

${ }^{12}$ Entrevista personal con el autor, el 9 de febrero de 2011. 
verría, "era cuestión de ser el primero en contar algo. No ahora. Hoy nosotros queremos ser la referencia, ofrecer información completa, más que ser sólo los primeros. Por supuesto queremos seguir siendo los primeros porque queremos ganar todas las batallas, pero esto es algo más que eso".

Dirigiéndose nuevamente a sus compañeros de la mesa, Echeverría les pregunta "¿por qué no llevamos esta noticia del congresista americano?", mientras muestra en su i-Pad la portada de elmundo.es. Bernie, el redactor responsable de los contenidos de la portada en ese momento, dando la impresión de que se esperaba una pregunta de ese tipo, contesta que "es muy tarde ahora en Washington y el corresponsal debe de estar durmiendo". "Bien" añade Echeverría, "si no podemos dar una información mejor, esperaremo"”.

Poco después, Echeverría regresa con la portada de elmundo.es en su i-Pad y pregunta "¿por qué no estamos contando que Bono está viajando a Guinea? 13". Bernie, un poco incómodo, le contesta en voz un poco más baja que "me dijeron que mejor lo ignorara". "¿Cómo? ¿Quién dijo esto? Está en todos los medios," añade Echeverría. "Pregúntale a..." Con un poco más de discreción, Echeverría se marcha a otro lugar de la redacción. Al cabo de unos minutos, Bernie recibe un texto discreto a publicar en el digital, en el que se explica lo mismo que es ya ampliamente conocido por los demás medios.

"Mire esto", comenta indignado Luis Sanz, responsable de coordinación de noticias nacionales en Efe, mostrando la agenda de actos diarios para el día. "Nosotros cubrimos estos eventos porque todos los demás medios lo harán. Y cada uno de los demás lo hará por el mismo motivo. Son eventos creados con los medios de comunicación en mente. Al final no hacemos más que seguirnos los unos a los otros", dice Sanz, quien está claramente en contra de esta nueva práctica en la que opina que los medios de comunicación están atrapados. La velocidad, la falta de reflexión por parte de los periodistas, el hecho de que todo puede ser noticia, en la medida en que todos los medios lo crean así, es la razón. "Todo el mundo corre a cubrir estas noticias y como [...] todos copiamos a los demás, temerosos de perdernos algo, decimos cosas de tan escaso valor que se olvidan horas más tarde. Son historias que mueren casi de inmediato. Y que las fuentes crean para tener la oportunidad de intervenir en la evolución diaria de la agenda", que se configura minuto a minuto en los medios digitales.

"Yo soy consciente de que historias que están en la red y a las que nadie atiende, si las cojo y las presento adecuadamente, desde un ángulo que tenga interés, serán copiadas por muchos otros medios. Sé que nuestro enfoque es importante por eso", dice Martín, entre satisfecho por ser copiado y frustrado porque le cuesta ver que su originalidad será de muchos en poco tiempo. Palabras más o menos idénticas tiene Casqueiro preguntado sobre el papel del periódico en el entorno digital. "Hemos detectado que somos seguidos [por otros]. Cuando decidimos un enfoque sobre un tema, o sobre una entrevista, inmediatamente vemos que ese enfoque lo han seguido otros medios. Pero nosotros queremos marcar la agenda también en la web. La velocidad genera un

${ }^{13}$ José Bono, Presidente del Congreso de los Diputados, estaba en esos momentos volando a Guinea Ecuatorial, para mantener allí varias entrevistas, según publicaba el periódico rival. 
riesgo mayor, de que no haya una perfecta sintonía entre el enfoque online con el que va a ir después en el periódico."

La tendencia a la homogeneidad, a publicar el mismo contenido que cualquier otro medio, se aplica a las noticias que los medios desean publicar pero también a aquellas que rechazarían, que preferirían ignorar. Los periodistas saben que su medio está siendo permanentemente monitorizado por sus colegas, para ver qué noticias publican, de forma que no incluir un tema que está en la agenda compartida es revelador a los ojos de los competidores de un interés en ocultar algo. Todos los periodistas del campo tienen una idea de los temas, las cuestiones y los intereses de sus colegas, tanto en el sentido de lo que sí les importa, como de lo que no. En todas las redacciones, incluso a nivel personal, la información sobre las preferencias ajenas circula ampliamente. Una información que también crea o destruye capital, crea o retira reconocimiento. Este seguimiento del competidor sucedía en el papel -el estudio clásico de Galtung y Ruge (1965) lo prueba, Bourdieu lo describe como 'juego de espejos' (1998/1996: 24) - pero la red ha exacerbado la monitorización y, por ende, la homogeneización. Hoy es imposible decir que no se conocía algo que circula en la red, por lo que los silencios son más llamativos.

Puesta esta dinámica de imitación bajo el paraguas de la interpretación sociológica de Bourdieu, el campo profesional y la dinámica interna del grupo juegan un papel diferente: los periodistas, temerosos de que, debido a la velocidad de la red se les haya escapado algo valioso que los competidores sí incluyen, tienden a repetir los contenidos que ven en otros lugares, a cubrir las mismas informaciones, a asegurarse que si no aportan nada nuevo, por lo menos están al mismo nivel que los demás. Mientras la exclusiva pesa menos, se intenta evitar lo opuesto a la exclusiva: ser el único medio al que se le ha escapado algo.

\section{Nuevos valores}

Pese a que desde 1994 (Díaz Noci, 2005), cuando los periódicos en papel iniciaron su carrera para estar presente en la red, han pasado casi 20 años, este y otros estudios en medios periodísticos digitales siguen describiendo la percepción general de estar aún asimilando y entendiendo cómo funcionan las dinámicas online. Una larga transición desde un modelo tradicional, en el que se conocían los valores, cómo se creaba prestigio, capital cultural, qué componía el habitus profesional, a otro en el que las piezas todavía no se han definido ni fijado con suficiente firmeza. Møller-Hartley (2011) señala que los nuevos valores aún no están bien definidos porque en el conjunto del campo los periodistas del papel tienen todavía un peso importante y son ellos los que aún conservan un papel relevante en la conformación del habitus colectivo. Por lo tanto, estamos en un momento confuso porque algunos referentes como la noticia exclusiva pierde peso pero aún sigue siendo valorada por quienes están en el papel y que conservan poder en el campo. No sabemos qué reemplazará a la exclusiva, ni siquiera si esas exclusivas con una vida de minutos tendrán valor. La incertidumbre es evidente en las redacciones, como revelan las tensiones internas derivadas de órdenes de empresa contrarias al habitus credo.

Usando las claves interpretativas de Bourdieu, vemos que el periodista ha entrado en la red con una idea de la exclusiva propia del medio impreso de la noticia; vemos 
que la práctica está enseñándoles que esta referencia en la red tiene un valor mínimo, si es que se aprecia. Pero, la convivencia del papel, aún muy potente en cuanto a referencias, con Internet, mantiene vivo un marco de valores que no termina de coincidir con las necesidades ni con las valoraciones del público.

Para los periodistas Internet significa cambios, impactos en su cultura profesional. Aunque no hay críticas a la tecnología, sí hay alarma sobre sus implicaciones. Raúl Casado, jefe de la sección de Sociedad y Futuro de Efe, un periodista de mediana edad, resume su actitud en pocas palabras: "Estoy viejo para estos cambios", sugiriendo no que su edad sea elevada sino que él, aferrado más a las prácticas tradicionales, tiene dificultades para adaptarse. Casado hacía esta reflexión tras explicar que el fin de semana anterior tuvo que cubrir una noticia y, en contra de las directrices de su empresa, acudió allí con su libreta de notas. "Si tuviera que haber empleado una cámara, no habría podido hacer mi trabajo bien"14.

Obsérvese cómo la famosa figura del gatekeeper (White, 1950; McCombs \& Shaw, 1972), el periodista todopoderoso que decide qué se publica y qué no, convirtiendo aquello en objeto de reflexión social, y condenando esto al ostracismo y la ignorancia, pierde relevancia debido a que la inmediatez permite que todos observen al mismo tiempo lo que constituye una especie de agenda pública de la cual al medio de comunicación le es imposible excluir tema alguno sin quedar en la evidencia de estar aplicando una censura que inevitablemente se volverá en su contra.

Es la lógica de la exclusiva, que todo el mundo pretende copiar, pero al revés: el medio que no quisiera publicar algo porque es un asunto que no encaja con sus inquietudes e intereses, tiene que hacerlo, so pena de que su desinterés quede en evidencia. ¿Cómo se puede explicar que la noticia de este viaje, disponible en todas las portadas de los digitales, no sea reproducida en un medio? La exclusiva crea prestigio según los valores del periodismo, pese que está perdiendo valor; pero las omisiones también afectan al capital, esta vez en negativo, dado que ahora la velocidad de la red convierte en inverosímil el argumento de no haberse enterado de la noticia.

\section{Conclusiones}

La retrasada adopción de las características de Internet al periodismo electrónico supone el reciente descubrimiento de que algunas prácticas y valores tradicionales, profundamente arraigados en el habitus del periodista, se transforman hasta el punto de perder sentido. Entre ellas, una de las prácticas que más apasiona a los profesionales, la búsqueda de la noticia exclusiva, se convierte en un fenómeno tan efímero que probablemente carece de valor para las audiencias y sea incluso de difícil valoración por parte de los profesionales. Curiosamente, la velocidad de la red hace que la copia, una práctica desprestigiada en el periodismo tradicional, normalmente condenada dentro de las redacciones, se convierta en una realidad diaria, creando una especie de 'antiexclusiva' que garantiza a los agentes de este campo una seguridad mínima.

${ }^{14}$ La dirección de $E f e$ ha pedido a su plantilla que utilice cámaras para cubrir sus noticias. La decisión fue recurrida a los tribunales, que dieron la razón a la dirección, pese a lo cual, en la mayor parte de los casos, los periodistas de la sede central de Madrid no las emplean. 
Estas prácticas conforman y son conformadas en cada campo. Por eso los integrantes del campo las aceptan, porque siempre ha sido así y ellos las incorporan desde el sentido común (Willig, 2013). La práctica de la noticia exclusiva tenía todas las características para arraigarse en la cultura del papel: tenía una vida como mínimo de 24 horas (aunque hemos visto el caso de un periodista que, buscando las noticias antes de un festivo, lograba incrementar su duración), lo cual impregnó el habitus profesional, convirtiéndose en una fuente de reconocimiento y, por tanto, de obtención de capital. Es una práctica que no se forma ante la audiencia sino ante los colegas, en el "juego de espejos" que describía Bourdieu (Bourdieu, 1998/1996).

En los primeros estudios sobre la red, la velocidad que aportaba parecía encajar con el habitus profesional, dado que el periodismo presume de ser veloz en contar lo que sabe (Domingo, 2006, 510-511). Sin embargo, en la realidad, tras más de una década, parece que estamos asistiendo a una consecuencia no prevista, que es la copia, la imitación o, incluso, el plagio (Phillips, 2011), que reduce la vida de la noticia exclusiva incluso hasta sólo unos minutos.

Una nueva doxa periodística parece estar surgiendo lentamente, resultado de la colisión entre dos formas de hacer periodismo. Según Bourdieu, los valores en un campo están, evolucionan y se reemplazan. Como el campo requiere de valores con los que medir el capital de sus miembros, la desaparición probable de la noticia exclusiva como elemento de prestigio, será reemplazada por otras formas de reconocimiento, quizás aún no configuradas suficientemente. Luis Gualdoni propone que en lugar de que el periodismo digital pretenda ser más veloz que los rivales, busque ser más profundo, más integral. "Yo no estoy de acuerdo con la política de mi periódico que dice que la portada debe ser modificada cada rato. Debe ser cambiada si hay noticias nuevas, pero no deberíamos tener una meta prefijada. ¿Piden los lectores tener una portada diferente cada vez que acceden a la web? Enseñémosles que cuando no sucede nada nuevo, la portada no cambia".

\section{Referencias bibliográficas}

ALTHEIDE, David (1976): Creating Reality: How TV News Distort Events. Beverly Hills (CA), Sage.

ALTHEIDE, David (1984):“A failure of perspective”. The Public Opinion Quarterly, Vol. 48, No. 2: 476-490.

ALTHEIDE, David (1997): "The News Media, The Problem Frame and the Production of Fear". The Sociological Quarterly. Vol. 38, No. 4: 647-668

ALTHEIDE, David (2004): "Media Logic and Political Communication". Political Communication 21 (3): 293-296

ANDERSON, Benedict (1993): Imagined Communities. London, New York, Verso

BOCZKOWSKI, Pablo (2004): Digitizing the News; Innovation in On-line Newspapers. Boston, Massachusetts, Institute of Technology.

BOCZKOWSKI, Pablo (2010): News at work. Imitation in an Age of Information Abundance. Chicago, University of Chicago Press. 
BOURDIEU, Pierre (1993): The field of cultural production. Nueva York, Columbia University Press.

BOURDIEU, Pierre (1996/1992): The Rules or Art. Genesis and Structure of the Literaty Field. Trans. Susan Emanuel. Stanford, Stanford University Press.

BOURDIEU, Pierre (1998/1996): On television. Trans. Priscilla Parkhusrt Ferguson. New York, New Press.

BOURDIEU, Pierre (2005): "The political field, the social science field, and the journalistic field", en BENSON, RODNEY y Neveu, Erik (eds.): Bourdieu and the Journalistic Field. Cambridge, Polity Press.

BOYD-BARRET, Oliver \& RANTANEN, Terhi (1996): The Globalization of News. London, Sage.

CAREY, James (1997/1969): “The Communications Revolution and the Professional Communicator”. en MUNSON E. S. \& WARREN C. A. (eds.): James Carey: A Critical Reader. Minneapolis, University of Minnesota Press.

DEUZE, Mark (2004): "What is multimedia journalism". Journalism Studies. 5: 2: $139-152$

DÍAZ NOCI, Javier (2001): “¿Hacia dónde va el periodismo en Internet?” Esfera Pública. Revista de Ciencias Sociales y de la Comunicación. 1: 77-100.

DÍAZ NOCI, Javier (2005): "Historia de los Cibermedios en España", en SALAVERRÍA, Ramón (ed.): Cibermedios, el impacto de Internet en los medios de comunicación en España. Sevilla, Comunicación Social, ediciones y publicaciones.

DODSON, Leslie \& STERLING, S. Revi (2012): "Ethics of participation: research or reporting?" The Electronic Journal on Information Systems in Developing Countries. 50: 6: 1-14.

DOMINGO, David (2006): Inventing Journalism Online: development of the Internet as a news medium in four Catalan newsrooms. Tesis doctoral, Universitat Autònoma de Barcelona, http://www.tesisenxarxa.net/TESIS_UAB/AVAILABLE /TDX-1219106-153347//dd1de1.pdf [acceso el 12 de abril de 2011].

FRANKLIN, Bob; HAMER, Martin; HANN, Mark; KINSEY, Marie \& RICHARDSON, John (2005): Key Concepts in Journalism Studies. London, Sage.

GALTUNG, Johan \& RUGE, Mari Holmboe (1965): "The structure of Foreign News: The presentation of the Congo, Cuba and Cyprus Crises in Four Norwegian Newspapers". Journal of Peace Research. 2: 64.

GÁMIR, Agustín (2005): "La industria cultural y los grupos multimedia en España, estructura y pautas de distribución territorial". Anales de geografia. 25: 179-202.

GARCÍA AVILÉS, José Alberto y CARVAJAL, Miguel (2008): “Integrated and CrossMedia Newsroom Convergence. Two Models of Multimedia News Production The Cases of Novotécnica and La Verdad Multimedia in Spain". Convergence. 14: 2: $221-230$ 
GASHER, Mike \& GABRIELE, Sandra (2004): "Increasing circulation? A comparative news-flow study of the Montrel Gazette's hard-copy and on-line editions". Journalism Studies. 5: 3: 311-323.

GÓMEZ, Bernardo y PANIAGUA, Francisco (2005): "Las ediciones digitales de los diarios españoles. Nacimiento y consolidación de un sector en auge". Razón y Palabra. Octubre, Noviembre. 47.

GUALLAR DELGADO, Javier (2007): "Prensa digital en 2006". Anuario ThinkEPI. 106-113.

HERMIDA, Alfred \& THURMAN, Neil (2008):"A clash of cultures". Journalism Practice. 2: 3: 343-356.

JONES, Janet \& SALTER, Lee (2012): Digital Journalism. London, Sage.

LÓPEZ HIDALGO, Antonio y MELLADO RUIZ, Claudia (2006): "Periodistas atrapados en la Red: Rutinas y situación laboral". Estudios sobre el Mensaje Periodístico. 12: 161-170. Madrid, Servicio de Publicaciones de la Universidad Complutense.

MARR, Andrew (2004): My Trade. McMillan, Londres.

MCCOMBS, Malcomb \& SHAW, Donald (1972): "The Agenda-Setting function of mass media". The Public Opinion Quarterly. 36: 2: 176-187.

MØLLER-HARTLEY, Jannie (2011): Radikalisering af Kampzonen. PhD. Roskilde, Denmark, Roskilde Universitet.

MOLOTCH, Harvey \& LESTER, Marilyn (1974): "News as Purposive Behavior: On the Stategic Use of Routine Events, Accidents and Scandals". American Sociological Review. 39: 1: 101-112.

MOLOTCH, Harvey \& LESTER, Marilyn (1975): “Accidental News: The Great Oil Spill as Local Occurrence and National Event". The American Journal of Sociology. 81: 2: 235-260.

NGUYEN, An (2008): "Facing the fabulous Monster". Journalism Studies 9: 1: 91-104

NGUYEN, An (2010): "Harnessing the potential of online news: suggestions from a study on the relationship between online news advantages and its post-adoption consequences". Journalism. 11: 2: 223-241

PATERSON, Chris (2008): "Introduction: Why Ethnography", en PATERSON, Chris \& DOMINGO, David (eds., 2008): Making Online News. The Ethnography of New Media Production. New York, Peter Lang.

POSTMAN, Neil (2000):"The Humanism of Media Ecology". Proceedings of the Media Ecology Association. Vbl. 1.

OJD (2012): "Informe sobre usuarios de Internet on line en España". Disponible en www.ojd.es/interactive [acceso el 25 de enero de 2012]

OLMOS, Víctor (1997): Historia de la Agencia Efe. El mundo en español. Madrid, Espasa Calpe. 
PHILLIPS, Angela (2010):“Transparency and the new ethics of journalism". Journalism Practice. 4: 3: 373-382.

ROBINSON, Sue (2011): "Convergence Crisis: News Work and News Space in the digitally transforming newsroom". Journal of Communication. 61: 1122-1141.

SALWEN, Michael (2001): "Evelyn Waugh's Scoop: The Facts Behind the Fiction". Journalism \& Mass Communication Quarterly. 78: 150.

SANCHA, David (2005): "El uso de la información de agencias en las ediciones electrónicas de los diarios en España". Athenea Digital. 8: 1-34

SCHLESINGER, Philip (1987): Putting 'reality'together. BBC News. London, Routledge.

STEENSEN, Steen (2010): "Online journalism and the promises of new technology". Journalism Studies. iFirst Article, 1-17

SILVA ECHETO, Víctor Manuel y BROWNE SARTORI, Rodrigo (2008): "Comunicación, Violencia y Poder Simbólico en la Sociología de Pierre Bourdieu”. Nómadas. Revista Crítica de Ciencias Sociales y Jurídicas. 1: 17

TUCHMAN, Gaye (1972): “Objectivity as Strategic Ritual: An Examination of Newsmen's Notions of Objectivity". The American Journal Of Sociology. 77: 7: 660679.

TUCHMAN, Gaye (1974): "Making news by doing work: routinizing the unexpected". American Journal of Sociology. 79: 110-131.

TUCHMAN, Gaye (1978): Making news: a study in the construction of reality. New York, Free Press.

WARNER, Malcolm (1970): "Decision-making in Network Television News" en TUNSTALL, Jeremy (ed.): Media Sociology: a Reader. Chicago, University of Illinois Press.

WARNER, Malcolm (1971): "Organizational context and control of policy in the television newsroom". British Journal of Sociology. 22: 3: 283.

WAUGH, Evelyn (1964): Scoop. London, Chapman \& Hall.

WHITE, David Manning (1950): "The gatekeeper: A case study in the selection of news". Journalism Quarterly, 27: 3: 383-396.

WILLIG, Ida (2013): "Newsroom ethnography in a field perspective". Journalism. 14: $3: 372$.

ZELIZER, Barbie (2004): Taking Journalism Seriously. London, Sage. 